\title{
Restricciones de la aplicación del principio de sustituibilidad de los idénticos salva veritate en Leibniz
}

Oscar M. Esquisabel

omesqui1@speedy.com.ar

Universidad Nacional de La Plata/ Universidad Nacional de Quilmes, Buenos Aires, Argentina/ CONICET

resumen 0 princípio de intersubstituição dos idênticos salva veritate, que constitui uma peça de importância central para a teoria leibniziana da demostração, para não falar de suas implicações ontológicas, recebeu a crítica de que encerra uma confusão entre uso e menção. Em contraste com essa crítica, o presente trabalho defende a tese de que 0 princípio não está suscetível a essa pretensa confusão, utilizando, para tanto, a distinção leibniziana entre "a consideração do modo de conceber" e a "consideração da coisa mesma". A introdução dessa distinção, porém, vem acompanhada com a necessidade de estabelecer restrições à aplicação do mencionado princípio.

palabras claves Leibniz; lógica; demostración; sustituibilidad; extensión; intensión

Se conoce la importancia que los principios poseen en la filosofia de Leibniz. Uno de los principios más importantes y, al mismo tiempo, más debatidos, ha sido el principio de sustituibilidad de los idénticos (PSI) salva veritate, del cual depende gran parte de la teoría leibniziana de la demostración. Asimismo, las versiones "ontológicas" de este principio, especialmente en conexión con su converso, el principio de identidad de los indiscernibles, poseen amplios alcances en la metafísica y, al decir de Leibniz, también en la física. Sin embargo, no nos ocuparemos en el presente contexto del problema de la relación entre la versión lógica y la ontológica del principio en cuestión. Sea de ello lo que fuere, el principio de sustituibilidad de los idénticos salva veritate aparece en un gran número de textos, especialmente de carácter lógico, con 
variaciones textuales más o menos importantes. La enunciación más usual es la siguiente:

PSI: Idéntico es aquello que puede sustituirse salva veritate en lugar de otro en todas sus instancias. Por ejemplo, equilátero equiángulo cuadrángulo, equilátero rectángulo cuadrilátero y finalmente cuadrado, son idénticos los tres y pueden sustituirse uno en lugar del otro (LEIBNIZ, 1923,VI 4 275). ${ }^{1}$

Este principio puede ser concebido como una definición de la identidad, aunque no es la única manera en que Leibniz la caracteriza. ${ }^{2}$ Sea como fuere, es claro que Leibniz se refiere a la mutua sustituibilidad salva veritate como una propiedad lógica fundamental de la identidad. Por otra parte, en algunas ocasiones Leibniz sustituye la condición de sustituibilidad en todas las instancias (ubique) por una formulación algo diferente, a saber, la sustituibilidad en cualquiera (ubivis) de las instancias. Tal modificación tiene su razón de ser en la demostración de ciertas propiedades de la identidad, como por ejemplo la simetría. La función del PSI en la teoría lebinziana de la demostración se sigue de la definición de la verdad proposicional como inclusión del predicado en el sujeto, para el caso de las proposiciones categóricas, y del consecuente en el antecedente, en el caso de las hipotéticas. Puesto que la demostración formal se funda en mostrar que la proposición a demostrar constituye una instancia del principio de identidad, se requiere de la regla del intercambio definicional para llevar a cabo esta reducción. En este sentido, el PSI opera como una regla de sustitución, ya que las definiciones pueden ser interpretadas como una identidad entre conceptos o términos. (LEIBNIZ, 1923,VI 4, p. 273-279)

No obstante la generalidad del uso que le concede Leibniz al mencionado principio, su aplicación ha recibido críticas, siendo la principal de ellas el hecho de que no queda claro entre qué cosas se establece la relación de identidad, de lo cual algunos de sus detractores, Quine, por ejemplo, extraen la consecuencia de que Leibniz confunde uso y mención. Esta clásica objeción se encuentra en Palabra y objeto:

Una análoga confusión de signo y objeto se manifiesta en Leibniz cuando explica la identidad como una relación entre los signos, no entre el objeto nombrado y él mismo: 'Eadem sunt quorum unum potest substituí alteri, salva veritate'. (QUINE, 1968, p. 128) 
También B. Mates retoma la crítica de Quine, a pesar de la defensa del principio que, entre otros, ha presentado Hidé Ishiguro. Así, Ishiguro sostiene que el PSI no confunde uso y mención, sino que se refiere siempre a la identidad entre conceptos, no a las cosas a que los conceptos se remiten (o que los instancian) (ISHIGURO, 1972, p 32-33). No obstante, a pesar de la defensa de Ishiguro, Mates reafirma la crítica quineana:

Es usual puntualizar que las formulaciones leibnizianas de esta definición [el PSI] involucran una confusión de nombres con lo que ellos nombran. Presumiblemente, son los nombres los que pueden ser intercambiados, pero son las cosas nombradas las que son o no son idénticas. El nombre 'Paris' no es idéntico con el nombre 'Alejandro', y tampoco tiene sentido decir que el hombre Paris puede o no puede ser intercambiado con un componente de la proposición. (MATES, 1972, p 100; Cfr. MATES, 1986, p.124 y ss)

De acuerdo con Mates, no hay buenas estrategias para evitar la confusión leibniziana, que queda envuelta en las paradojas señaladas por Frege en Sinn und Bedeutung (MATES, 1986, p 128). No obstante, PSI puede salvarse mediante una formulación metalingüística en términos de mundos posibles (Id., p. 129.).

En el presente contexto, no analizaremos pormenorizadamente las interpretaciones de Mates, sostenidas por un abundante apoyo textual. Sin embargo, es posible indicar de qué modo puede sortearse la objeción basada en la confusión uso-mención a partir de la idea que ha defendido Ishiguro, a saber, que la identidad, en principio, se establece entre conceptos y no entre las cosas que caen bajo los conceptos, si es que esta forma de presentar la relación entre conceptos y objetos es válida para el caso de Leibniz. Así, se puede evitar una de las objeciones más claras al principio, a saber, la de que en una proposición (que es lo que está sugerido por la condición de salva veritate) no son cosas las que se intercambian, sino conceptos. Para ello, aceptaré o daré por sentado que, para Leibniz, el sentido de una proposición es 'composicional', es decir, dicho sentido se encuentra determinado por los conceptos que en ella intervienen. Del mismo modo, esos mismos conceptos son los que determinan sus condiciones de verdad.

El principio de solución ante la objeción que hemos mencionado recurre a una distinción que Leibniz introduce entre la "consideración de 
las cosas" y la "consideración de los modos de concebir". Como veremos, esta distinción es análoga a la diferencia entre sentido y denotación y su naturaleza es tal que restringe, en algunos casos, la aplicación de PSI a contextos de carácter estrictamente extensional, mientras que en otros casos, donde se afirma una identidad intensional entre conceptos (como en el caso de las definiciones), no hay en principio restricciones.

Uno de los motivos por el que es probable que se acuse a Leibniz de haber cometido la confusión uso-mención radica en el hecho de que da el nombre de 'términos' (termini) a las partes de la proposición. Si por término se entiende una entidad lingüística que forma parte de una oración, parece entonces sugerirse la idea de que, al hablar de términos intercambiables e idénticos, Leibniz incurre en la confusión entre uso y mención: ${ }^{3}$ no son los términos, sino las cosas designadas las que son idénticas, mientras que lo que se intercambia son entidades lingüísticas.

No es raro encontrar pasajes donde Leibniz presenta a los términos como entidades gramaticales o lingüísticas, ${ }^{4}$ pero la mayoría de las veces 'término' significa 'concepto', 'noción' o 'idea', como surge de la simple lectura de los textos relevantes. Así, por ejemplo, cuando se dice que un término A encierra o incluye otros términos C, D, etc., Leibniz se refiere claramente a conceptos y no a entidades lingüísticas (LEIBNIZ, 1923,VI 4, p. 552, cfr. A VI 4, p. 277, inter alia). Admitamos, por tanto, que en los contextos en los que Leibniz habla de términos, las entidades que se están mentando son conceptos, nociones o ideas. Siendo ello así, lo que intercambiamos cuando aplicamos el principio de sustituibilidad no son entidades gramaticales o nombres, sino conceptos. Desechamos la posibilidad de que lo que se intercambie sean cosas, ya que una cosa, por sí misma, no forma parte de una verdad, es decir, de una proposición. Las traducciones del principio, que suelen traducir los pronombres o adjetivos neutros por el término 'cosa', ocultan esta circunstancia. De esta manera, se desactiva la objeción de la confusión entre uso y mención.

En cualquier caso, podemos preguntarnos si esta respuesta no acarrea más problemas de los que soluciona. En efecto, si la relación de identidad se establece entre conceptos, no evitamos la objeción que se le aplicaba a la identidad de nombres, a saber, ¿cómo es posible que dos conceptos distintos sean idénticos? Después de todo, la aparente contradicción en el caso de los nombres recibe una rápida solución apelando a la identidad de 
la denotación: no es que sean idénticos los nombres, sino que lo son sus denotaciones. Sin embargo, en el caso de los conceptos (o términos), la cuestión es más grave: no se trata de meras entidades gramaticales (como en el caso de los nombres), sino que son los conceptos en cuanto tales los que se dicen idénticos, siendo al mismo tiempo diferentes.

Claramente, hay casos en los que esta contradicción es aparente. Se trata de aquellos conceptos que pueden ser exhaustivamente analizados en términos de sus conceptos (nociones, términos) componentes, de manera tal que dicho análisis se pueda expresar como una equivalencia definicional. En ese caso, tenemos una identidad que podemos denominar 'intensional' entre el definiens y el definiendum. Sin entrar en complicaciones más serias, podría decirse que se trata de uno y el mismo concepto presentado (o "pensado") de manera diferente: en un caso de manera simbólica o "ciega" (el definiendum) y, en el otro, de forma analítica o "intuitiva" (el definiens).

No obstante, hay otros casos que no admiten esta solución, ya que se trata de conceptos efectivamente diversos en lo que respecta a su contenido, aunque, por otra parte, son sustituibles recíprocamente salva veritate. Para estos casos, el PSI no siempre vale y, por tanto, debe restringirse su aplicación. Por ejemplo, es común que Leibniz apele a los conceptos de 'triángulo' y 'trilátero' como casos de sustituibilidad salva veritate (LEIBNIZ, 1923, VI 4, p. 283). Sin embargo, en los Nuevos Ensayos, Leibniz sostiene que dichos conceptos son idénticos en algunos respectos, pero no en todos:

...pues diciendo que el triángulo y el trilátero no son lo mismo estaría equivocado, ya que si lo consideramos cuidadosamente, encontramos que tres lados y tres ángulos van siempre juntos... Sin embargo, siempre se puede decir en abstracto que el triángulo no es el trilátero, o que las razones formales del triángulo y del trilátero no son las mismas, tal como los filósofos sostienen. Son respectos de una y la misma cosa (LEIBNIZ, 1923,VI 6, p. 363 (GPV 344), cfr. LEIBNIZ, 1923,VI 6, p. 408).

Encontramos un caso análogo en el siguiente pasaje, esta vez referido a conceptos en relación con individuos:

Si A es B y B es A, entonces A y B se dicen idénticos. Es decir, A y B son idénticos si pueden sustituirse entre sí en todas sus instancias (excepto 
en aquellos casos en los que se trata no de la cosa, sino del modo de concebir, por el que ciertamente difieren. Así, 'Pedro' y 'El Apóstol que negó a Cristo' son lo mismo, y un término puede ponerse en lugar del otro, excepto cuando considero ese mismo modo de concebir que algunos denominan reflexivo, por ejemplo, cuando digo 'Pedro, en tanto y en cuanto fue el Apóstol que negó a Cristo, pecó', no puedo sustituir de ningún modo 'Pedro', es decir, no puedo decir 'Pedro, en cuanto fue Pedro, pecó'. (LEIBNIZ, 1923,VI 4, p. 522)

En principio, puede causar perplejidad que presentemos este pasaje como un caso de sustitución conceptual salva veritate, ya que interviene el nombre de un individuo 'Pedro'. No hay que olvidar, sin embargo, que Leibniz admite conceptos individuales (que se expresan mediante nombres propios). Sea lo que fuere en lo que se refiere a la anterior observación, se produce en el ejemplo un fallo de la sustituibilidad salva veritate, que ya fuera destacado tanto por Mates (MATES, 1986, p 130-132) como por Angelelli (ANGELELLI, 1967). Ciertamente, se pueden señalar varios pasajes donde Leibniz señala este mismo fallo. ${ }^{6}$ En cualquier caso, interesa puntualizar en los ejemplos propuestos las razones por las que el PSI deja de aplicarse correctamente. Para ello, Leibniz recurre a la necesidad de establecer diferenciaciones entre tipos de proposición sobre la base del modo de su referencia. Así, hay proposiciones que se refieren o denotan estados de cosas u objetos, es decir, en palabras de Leibniz, "tratan de la cosa misma". En estos casos, el PSI se aplica sin problemas, por lo que 'Pedro' es recíprocamente sustituible con 'el Apóstol que negó a Cristo', así como ocurre lo mismo con 'triángulo' y 'trilátero'. En cambio, en oposición a las anteriores, que son "directas" (LEIBNIZ, 1903, p. 363), existe un segundo tipo de proposiciones, que Leibniz denomina "reflexivas", para las que el PSI no vale. En efecto, las proposiciones reflexivas son aquellas que involucran en sí mismas una cierta relación con la mente o espíritu que concibe. ${ }^{7}$ En ocasiones, Leibniz recurre a una caracterización que sugiere la idea contemporánea de propiedades metalingüísticas o metalógicas, aunque ciertamente las proposiciones que resultan se refieren a conceptos y no a objetos gramaticales, si bien Leibniz también atribuye carácter reflexivo a las proposiciones gramaticales en sentido estricto (LEIBNIZ, 1923,VI 4, p. 403). 
Leibniz denomina también "reduplicativas" a las proposiciones reflexivas (Id. ibid.), aunque en sentido propio las primeras son una subclase de las segundas. De acuerdo con una tradición que Leibniz retoma, las proposiciones reduplicativas son aquellas que se construyen con la partícula en cuanto que (qua, inquatum, quatenus... eatenus, prout) y tienen como efecto el que se hable, como adelantamos, no de la cosa misma, sino del modo de concebir, por lo que tienen como efecto una especie de abstracción. En otras palabras, las proposiciones reduplicativas aíslan las razones formales que hacen que un predicado se aplique a un determinado objeto. Propiamente hablando, son esas razones formales las que constituyen "modos de concebir", que, en cuanto tales, ya no pueden intercambiarse salva veritate. En síntesis, las proposiciones reflexivas efectúan un cambio de punto de vista en lo que respecta a la referencia, ya que hacen pasar de un contexto extensional, donde lo que cuenta son las denotaciones o extensiones de los respectivos conceptos (es decir, donde lo que interesa son los objetos como instancias de conceptos), a un contexto de carácter intensional, en el que se pone de relieve (y se hace referencia) al contenido propio del concepto o noción, que es así tanto intensional como intencional (en el sentido de que tiene como correlato un acto de concepción). Es justamente el contenido propio del concepto lo que lo diferencia de su idéntico en el contexto extensional. Así, las proposiciones reflexivas actúan imponiendo una especie de restricción acerca de la clase de proposiciones que se pueden realizar acerca del concepto aislado por la vía de la reduplicación.

En este sentido, lo que Leibniz denomina la "consideración de la cosa misma" o modo directo representa el contexto extensional, en el que los conceptos están tomados en su función directa de representar o de denotar los objetos mismos; por esa razón, son idénticos a partir de su coextensividad, a pesar de que su contenido sea diverso, como ocurre con ‘trilátero’ y ‘triángulo’ o ‘Pedro’ y ‘el Apóstol que negó a Cristo’. Así, en el caso de la sustituibilidad salva veritate, Leibniz parece privilegiar el hecho de que los conceptos denotan o se refieren a objetos, independientemente de que expresen diversos aspectos de uno y el mismo objeto (o de una y la misma clase de objetos) y es por ello que el punto de vista es de carácter extensional. De esta forma, los "aspectos" o conceptos coextensivos no son otra cosa que diversidades intencionales de acuerdo con las cuales una 
misma cosa se presenta a la mente y es por esa razón que se las relega a meros "modos de concebir" (LEIBNIZ, 1923, VI 4, p. 1499). Queda en pie la cuestión de si estos últimos son meras diversidades intencionales o si expresan propiedades realmente diferentes.

En todo caso, la situación cambia de manera fundamental cuando se pasa del modo directo al reflexivo, en el que se consideran los conceptos en su calidad de "modos de concebir" en cuanto tales. Como dijimos, pasamos del contexto extensional al intensional. En el contexto extensional, el contenido específico del concepto, lo que se denomina su "razón formal" o "formalidad", es el fundamento de su referencia o denotación. ${ }^{8}$ En el contexto intensional, tomado por sí mismo, esa misma razón formal constituye un modo de concebir. Así, si los modos de concebir eran, desde el punto de vista extensional, aspectos del objeto y por esa razón lo denotaban, en el punto de vista reflexivo se desconecta la relación de denotación, considerándose los conceptos pura y exclusivamente desde el punto de vista intensional, por lo que sólo se tienen en cuenta las relaciones intensionales que dicho concepto mantiene con otros conceptos. Tal parece ser la justificación de que 'triángulo' no pueda ser sustituido por 'trilátero' en la proposición “el triángulo, en cuanto tal, tiene 180 grados”, en la medida en que el concepto de 'grado' está intensionalmente conectado con el concepto de 'ángulo', pero no con el de 'lado'.

Así, la paradoja que habíamos planteado al principio respecto de la identidad de conceptos en sí mismos diversos recibe una solución a través de la diferencia entre las proposiciones directas y reflexivas o, si se quiere, entre el concepto como "razón formal" y "modo de concebir". La diferencia es la misma que media entre un contexto extensional y otro intensional. En el primero, se privilegia la denotación del concepto o noción, y por eso rige la sustituibilidad salva veritate para términos coextensivos, mientras que en el segundo, que es también intencional, se hace abstracción de la denotación y se tienen en cuenta solamente las relaciones intensionales y, por esa razón, es necesario imponer restricciones al PSI en sus formulaciones usuales. Por esta vía, la diferencia entre el 'modo directo' y el 'modo reflexivo' o entre la "consideración de las cosas mismas" y la "consideración del modo de concebir" se asemeja a la solución que da Frege al problema de la identidad por medio de la distinción entre sentido y denotación. Así, el "sentido" correspondería a lo que Leibniz denomina "modo de 
concebir", que se expresa en las proposiciones reduplicativas, mientras que la "denotación" coincidiría con "la consideración de la cosa misma".

Para concluir, de nuestro análisis resulta la posibilidad de sostener dos aplicaciones básicas del PSI. La primera carece en principio de problemas y no parece estar sometida a restricciones: se trata de la aplicación del PSI en el caso del intercambio definicional. Puesto que lo que se establece es la equivalencia intensional entre conceptos, el intercambio salva veritate entre definiens y definiendum no parece estar sometido a restricción alguna. En contraste, en el caso de conceptos que, aunque tengan la misma extensión, expresen diferentes "modos de concebir", el PSI valdría para Leibniz irrestrictamente en los contextos extensionales, pero su aplicación estaría limitada cuando se pasa a contextos intensionales y, más específicamente, en el caso de las proposiciones reflexivas, en las que rigen pura y exclusivamente relaciones de carácter intensional.

${ }^{1}$ Otros pasajes con formulaciones más o menos equivalentes: LEIBNIZ, 1923, VI 4, p. 552; LEIBNIZ, 1923,VI 4, p. 282; LEIBNIZ, 1923,VI 4 846, inter alia. En otras ocasiones, Leibniz designa a los idénticos en términos de 'coincidentes', por ejemplo, en LEIBNIZ, 1923,VI, p. 846.

2 MATES, 1972, p 99. En otras ocasiones, la define de esta manera: A es lo mismo que B si A es B y B es A. Cfr. LEIBNIZ, 1923,VI 4, p. 274, C, p. 53. Se entiende que el 'si' vale por un 'si y sólo si'.

${ }^{3}$ LEIBNIZ, 1923,VI 4, p. 867: "Idem sunt duo Termini, si alter alteri substituí potest salva veritate".

${ }^{4}$ Por ejemplo, LEIBNIZ, 1923,VI 4, p. 570, texto en el que se utiliza 'término' para referirse a entidades gramaticales tales como 'adjetivo' o 'sustantivo'. Cfr. LEIBNIZ, 1923, VI 4, p. 287.

${ }^{5}$ LEIBNIZ, 1923,VI 4, p. 288: "Per Terminum non intelligo nomen, sed conceptum seu id quod nomine significatur, possis et dicere notionem, ideam”.

${ }^{6}$ LEIBNIZ, 1923,VI 4, p. 401-403; A VI 4, p. 752; cfr. MATES, 1986, p 127 y 131. C, p. 261: "A = B significat A et B esse idem, seu ubique sibi posse substituí (Nisi prohibeatur, quod fin in iis, ubi terminus aliquis certo respectu considerari declaratur, v. g. licet trilaterum et triangulum sint idem, tamen si dicas triangulum, quatenus tale, habet 180 gradus; non potest substitui trilaterum. Est in eo aliquid materiale”. Citado también por MATES, 1986, p. 130.

${ }^{7}$ LEIBNIZ, 1923,VI 4, p. 656, referido a cuestiones gramaticales. Sin embargo, cfr. LEIBNIZ, 1923,VI 4, p. 403, donde lo reflexivo en el campo de lo gramatical se compara con lo reflexivo en el campo conceptual.

doispontos, Curitiba, São Carlos, vol. 11, n. 2, p.19-28, outubro, 2014 
${ }^{8}$ LEIBNIZ, 1923,VI 4, p. 752, cfr. LEIBNIZ, 1923, VI 6, p. 363. La razón formal se expresa como abstracto, por ejemplo, 'triangularidad' y 'trilateralidad'.

\section{Referências bibliográficas}

ANGELELLI, I. 1967. "On identity and interchangeability in Leibniz and Frege”, Notre Dame Journal of Formal Logic 8, pp. 94-100.

ISHIGURO, H. 1972. Leibniz's Philosophy of Logic and Language. Londres, Duckworth.

LEIBNIZ, Gottfreid Wilhelm. (C) 1903. Opuscules et fragments inédits, editados por Louis Couturat. Paris (repr. by Georg Olms Verlag, Hildesheim/New York. 1988). Citado como C.

LEIBNIZ, Gottfried Wilhelm. (A) 1923. Sämtliche Schriften und Briefe, editados por la Academia de Ciencias de Berlin desde 1923. Citado como A, seguido de serie, volumen y página.

MATES, B. 1972. "Individuals and modality in the philosophy of Leibniz”, Studia Leibnitiana 4, pp. 81-118. . 1986. The Philosophy of Leibniz. Metaphysics and Language. Oxford, Oxford University Press.

QUINE,W. O. 1968. Palabra y objeto. Trad. Manuel Sacristán. Barcelona, Labor. 